\title{
Brain stiffness increases with myelin content
}

\author{
J. Weickenmeier ${ }^{\mathrm{a}}$, R. De Rooija ${ }^{\mathrm{a}}$ S. Budday ${ }^{\mathrm{b}}$, P. Steinmann ${ }^{\mathrm{b}}$, T.C. Ovaert ${ }^{\mathrm{c}}$, E. Kuhl ${ }^{\mathrm{a}, \mathrm{d}, *}$ \\ ${ }^{a}$ Department of Mechanical Engineering, Stanford University, Stanford, CA 94305, USA \\ ${ }^{b}$ Department of Mechanical Engineering, University of Erlangen-Nuremberg, 91058 Erlangen, Germany \\ ${ }^{c}$ Department of Aerospace and Mechanical Engineering, The University of Notre Dame, Notre Dame, IN 46556, USA \\ ${ }^{d}$ Department of Bioengineering, Stanford University, Stanford, CA 94305, USA
}

\begin{abstract}
Brain stiffness plays an important role in neuronal development and disease, but reported stiffness values vary significantly for different species, for different brains, and even for different regions within the same brain. Despite extensive research throughout the past decade, the mechanistic origin of these stiffness variations remains elusive. Here we show that brain tissue stiffness is correlated to the underlying tissue microstructure and directly proportional to the local myelin content. In 116 indentation tests of six freshly harvested bovine brains, we found that the cerebral stiffnesses of $1.33 \pm 0.63 \mathrm{kPa}$ in white matter and $0.68 \pm 0.20 \mathrm{kPa}$ in gray matter were significantly different $(\mathrm{p}<0.01)$. Strikingly, while the interspecimen variation was rather moderate, the minimum and maximum cerebral white matter stiffnesses of $0.59 \pm 0.19$ $\mathrm{kPa}$ and $2.36 \pm 0.64 \mathrm{kPa}$ in each brain varied by a factor of four on average. To provide a mechanistic interpretation for this variation, we performed a histological characterization of the tested brain regions. We stained the samples with hematoxylin and eosin and luxol fast blue and quantified the local myelin content using image analysis. Interestingly, we found that the cerebral white matter stiffness increased with increasing myelin content, from $0.72 \mathrm{kPa}$ at a myelin content of $64 \%$ to $2.45 \mathrm{kPa}$ at a myelin content of $89 \%$, with a Pearson correlation coefficient of $\rho=0.91(\mathrm{p}<0.01)$. This direct correlation could have significant neurological implications. During development, our results could help explain why immature, incompletely myelinated brains are softer than mature, myelinated brains and more vulnerable to mechanical insult as evident, for example, in shaken baby syndrome. During demyelinating disease, our findings suggest to use stiffness alterations as clinical markers for demyelination to quantify the onset of disease progression, for example, in multiple sclerosis. Taken together, our study indicates that myelin might play a more important function than previously thought: It not only insulates signal propagation and improves electrical function of single axons, it also provides structural support and mechanical stiffness to the brain as a whole.
\end{abstract}

Keywords: Brain; mechanical testing; indentation; soft matter; stiffness; myelin

'Da das Bedürfniss, sie mit einem Worte bezeichnen zu können, vorliegt, so schlage ich vor, um jede Verwechselung mit anderen schon bezeichneten, aber noch problematischen Substanzen zu vermeiden, sie Markstoff, Myelin zu benennen.'

Rudolf Ludwig Carl Virchow [1854]

\section{Motivation}

Increasing evidence suggests that the mechanical environment of the brain plays an important role in neuronal development [1] and disease [2]. Magnetic resonance elastography now allows us to characterize this mechanical environment in vivo [3], and perform non-invasive, repeated measurements to quantify regional and temporal stiffness variations in the living brain [4]. Recent studies have successfully used magnetic resonance elastography to correlate alterations in brain stiffness to neurodegeneration in chronic neurological disorders [5] and in aging [6, 7]. However, the precise mechanistic origin of these stiffness variations is unknown.

\footnotetext{
* Corresponding author

Email address: ekuhl@stanford.edu (E. Kuhl)
}

Our brain consists of an outer gray matter layer made up of neuronal cell bodies, dendrites, and unmyelinated axons and an inner white matter core composed of oligodendrocytes, astrocytes, microglia, and a dense network of myelinated axons. The myelin sheath around nerve fibers was first discovered by Rudolf Virchow in 1854 and has since then been subject of extensive investigation [8]. We now know that myelin plays a critical role in insulating signal propagation and improving electrical function. Myelin consists of $40 \%$ water and its dry mass is composed of $70-85 \%$ lipids and $15-30 \%$ protein [9]. In human brain development, the first myelin sheaths form around week 14 of gestation; yet, the most progressive phase of myelination is aligned with an infant's rapid development during the first year of life. Dysmyelination, the defective formation of insulating myelin sheaths, is a manifestation of genetic mutations associated with leukodystrophies and also 
present in cases of schizophrenia [10]. Demyelination, the progressive loss of myelin sheaths, is a classical hallmark of several neurodegenerative autoimmune diseases including multiple sclerosis [11]. While the electrical effects of altered myelin concentrations are widely recognized, the mechanical effects of myelin remain poorly understood. This is the objective of the present study.

A robust, reliable, and reproducible method to characterize the mechanical behavior of the brain is indentation testing [12]. While tissue indentation is only rarely used to probe living brain in vivo [13], it is currently gaining popularity as a high-resolution, high-fidelity approach to probe individual different regions of gray and white matter tissue in vitro $[14,15]$. On the cellular level, atomic force microscopy indentation suggests that gray matter is about twice as stiff as white matter, both in mice [16] and rats [17]. On the tissue level, mechanical indentation tests reveal the opposite with gray matter being about one third softer than white matter, both in pigs [12, 18] and sheep [14]. On the whole organ level, magnetic resonance elastography suggests that gray and white matter are rather indistinguishable in vivo, both in ferrets [4] and humans [19]. Discrepancies in these measurements not only reflect the extreme strain rate sensitivity of brain tissue, but also its non-linear behavior [15, 20] and its compression stiffening [21]. This study seeks to unravel the ongoing controversy between gray and white matter stiffness measurements.

\section{Materials and methods}

Our experimental study combined mechanical characterization via indentation testing and histological characterization via staining to correlate mechanical parameters to tissue microstructure. We analyzed six fresh bovine brains collected from a local slaughterhouse (Martin's Custom Butchering, Wakarusa, IN). Fig. 1 illustrates a representative sagittal brain slice prepared for mechanical and histological characterization.

\subsection{Mechanical characterization}

Within two hours post mortem, we prepared 5mmthick sagittal slices with a consistent offset relative to the midline using a custom-designed slicing tool, placed them in a $100 \mathrm{~mm}$-diameter petri dish, and mounted the dish into a Hysitron TI 950 TriboIndenter ${ }^{\mathrm{TM}}$ (Hysitron Inc., Eden Prairie, MN) with an xZ 500 extended displacement stage [14]. This setup allows for indenter tip displacements of up to $500 \mu \mathrm{m}$ with a $1 \mathrm{~nm}$ displacement control precision and a force measurement resolution of $<0.1 \mathrm{nN}$. We performed all measurements at room temperature, under displacement control, using a custom-made $1.5 \mathrm{~mm}$ diameter flat punch indenter $[22,23]$. We stabilized each indentation region with a $12 \mathrm{~mm}$-diameter stainless steel washer and hydrated the tissue surface inside the washer with phosphate-buffered saline solution to prevent specimen dehydration and minimize adhesion. fresh sagittal slice

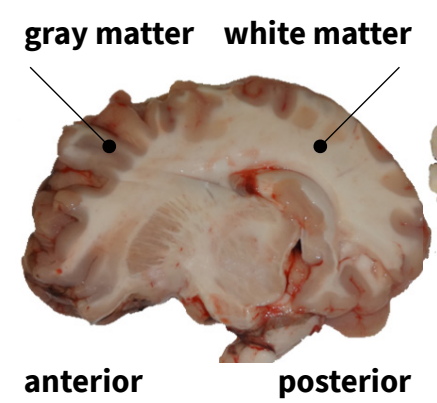

$1 \mathrm{~cm}$

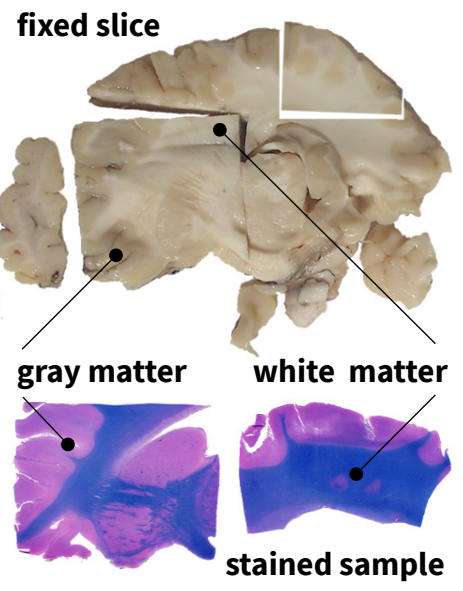

Figure 1: Sagittal slice prepared for mechanical and histological characterization. Freshly harvested slice for mechanical testing (left), same sample fixed in $10 \%$ formalin following mechanical testing (top right), and two $4 \mathrm{~cm} \times 4 \mathrm{~cm}$ sections prepared for histology using a principal hematoxylin \& eosin (H\&E) and luxol fast blue (LFB) stain (bottom right).

Fig. 2 illustrates a characteristic force-displacement curve of our mechanical characterization. We prescribed a trapezoidal loading-holding-unloading profile with a maximum indenter displacement of $400 \mu \mathrm{m}$ at a loading and unloading rate of $5 \mu \mathrm{m} / \mathrm{s}$ and a holding time at maximum indentation of $10 \mathrm{~s}$ [14]. Upon loading, the indentation force increased gradually. During the short holding period, the force decreased as the tissue stress relaxed. Upon unload-

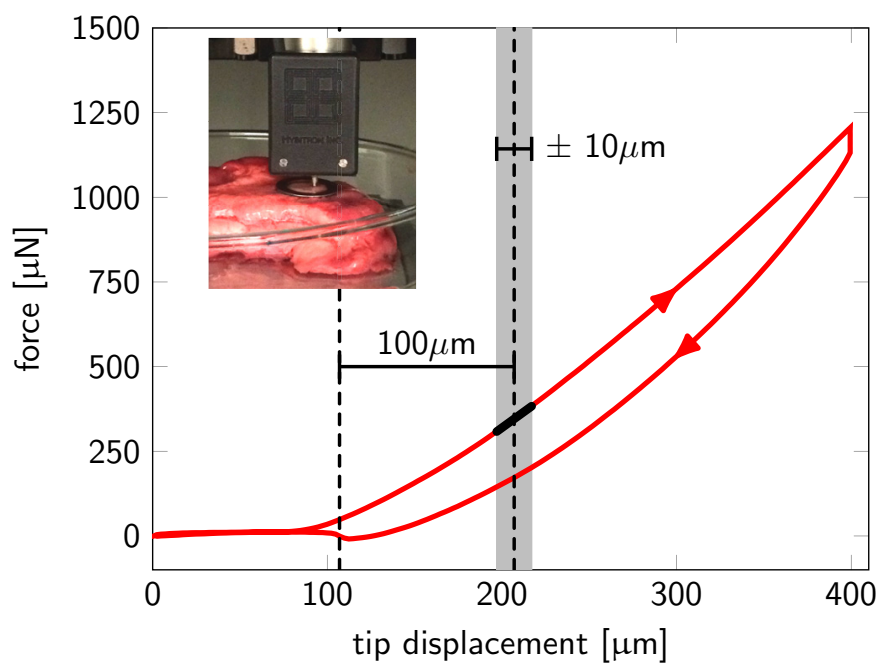

Figure 2: Characteristic force-displacement behavior under displacement control. We prescribed a trapezoidal loading-holding-unloading profile with a maximum indenter displacement of $400 \mu \mathrm{m}$, at a loading and unloading rate of $5 \mu \mathrm{m} / \mathrm{s}$, and a holding time at maximum indentation of $10 \mathrm{~s}$. We automatically detected the indenter-sample separation point upon unloading, and consistently zeroed all force and displacement measurements retrospectively with respect to this point (dashed line). We interpreted the mean slope at $100 \pm 10 \mu \mathrm{m}$ of the loading curve as the contact stiffness $k$ of each measurement (black line in gray box). 
ing, the indentation force decreased, became negative as the indentor tip was pulled down by tissue adhesion, and returned to its initial value upon complete separation of tip and sample. We automatically detected the separation point during unloading, and consistently zeroed all force and displacement measurements retrospectively with respect to this point. We interpreted the mean slope at $100 \pm 10 \mu \mathrm{m}$ of the loading curve as the contact stiffness $k$ of each measurement. For this contact stiffness, we determined the effective elastic modulus [24],

$$
E_{\mathrm{eff}}=\frac{\sqrt{\pi} k}{2 \sqrt{A}},
$$

where $A=1 / 4 \pi d^{2}$ is the cross-sectional area of the flat punch with diameter $d$. The effective modulus generally accounts for the elastic deformation in both sample and indenter,

$$
\frac{1}{E_{\mathrm{eff}}}=\frac{1-\nu_{\mathrm{smp}}^{2}}{E_{\mathrm{smp}}}+\frac{1-\nu_{\mathrm{ind}}^{2}}{E_{\mathrm{ind}}},
$$

where $E_{\text {smp }}$ and $E_{\text {ind }}$ are the Young's moduli and $\nu_{\text {smp }}$ and $\nu_{\text {ind }}$ are the Poisson's ratios of the sample and the indenter. Assuming that the stiffness of the indenter is orders of magnitudes larger than the sample, $E_{\text {ind }} \gg E_{\text {smp }}$, and that the tissue is nearly incompressible, $\nu_{\mathrm{smp}} \approx 0.5$, we obtained the following linear relation between the sample stiffness $E_{\mathrm{smp}}$ and the contact stiffness $k$,

$$
E_{\mathrm{smp}}=\left[1-\nu_{\mathrm{smp}}^{2}\right] E_{\mathrm{eff}}=\frac{3}{4} \frac{k}{d}
$$

We tested six different mammalian brains and performed a total of $n=116$ indentation tests. For each brain, we performed three measurements in cerebral gray matter and in cerebellar white matter, resulting in a total of $n=18$ measurements each. In addition, we performed a series of 12 to 18 white matter measurements along the anteriorposterior arch as illustrated in Fig. 5, resulting in a total of $n=80$ measurements in cerebral white matter. We did not test cerebellar gray matter because those regions were too small for our current test setup and indenter diameter.

\subsection{Histological characterization}

Following mechanical testing we prepared three randomly selected samples for light microscopy. We fixed the slices using $10 \%$ buffered formalin of $4 \%$ formaldehyde in phosphate buffered saline solution. We cut each slice into $4 \mathrm{~cm} \times 4 \mathrm{~cm}$ samples, gradually dehydrated the samples by replacing tissue water by alcohol, cleared the samples by replacing alcohol by xylene, and embedded the dehydrated samples in paraffin wax blocks. From these, we prepared 8-10 nm thick histological slices using a microtome. We stained the slices using hematoxylin and eosin (H\&E), where hematoxylin colors cell nuclei in blue and a subsequent counterstaining with eosin colors eosinophilic intracellular and extracellular protein structures in shades of red, pink, and orange. We further stained the slices using luxol fast blue (LFB), where myelin appears in blue, neuropil in pink, and nerve cells in purple. At the macroscopic scale, combined hematoxylin and eosin and luxol fast blue staining allowed us to differentiate between gray and white matter tissue; at the microscopic scale, it allowed us to characterize the myelin content in white matter tissue. Using the image analysis tools of MATLAB, we quantified the relative area fraction of the red- and bluecolored regions in the combined stain, and interpreted the blue-colored area fraction as the local myelin content.

\section{Results}

\subsection{Mechanical characterization}

Fig. 3 summarizes the stiffnesses of different tissue types for six brains and a total of $n=116$ indentation tests. We tested a total of $n=80$ samples of cerebral white matter, $n=18$ samples of cerebral gray matter, and $n=18$ samples of cerebellar white matter. Individual measurements
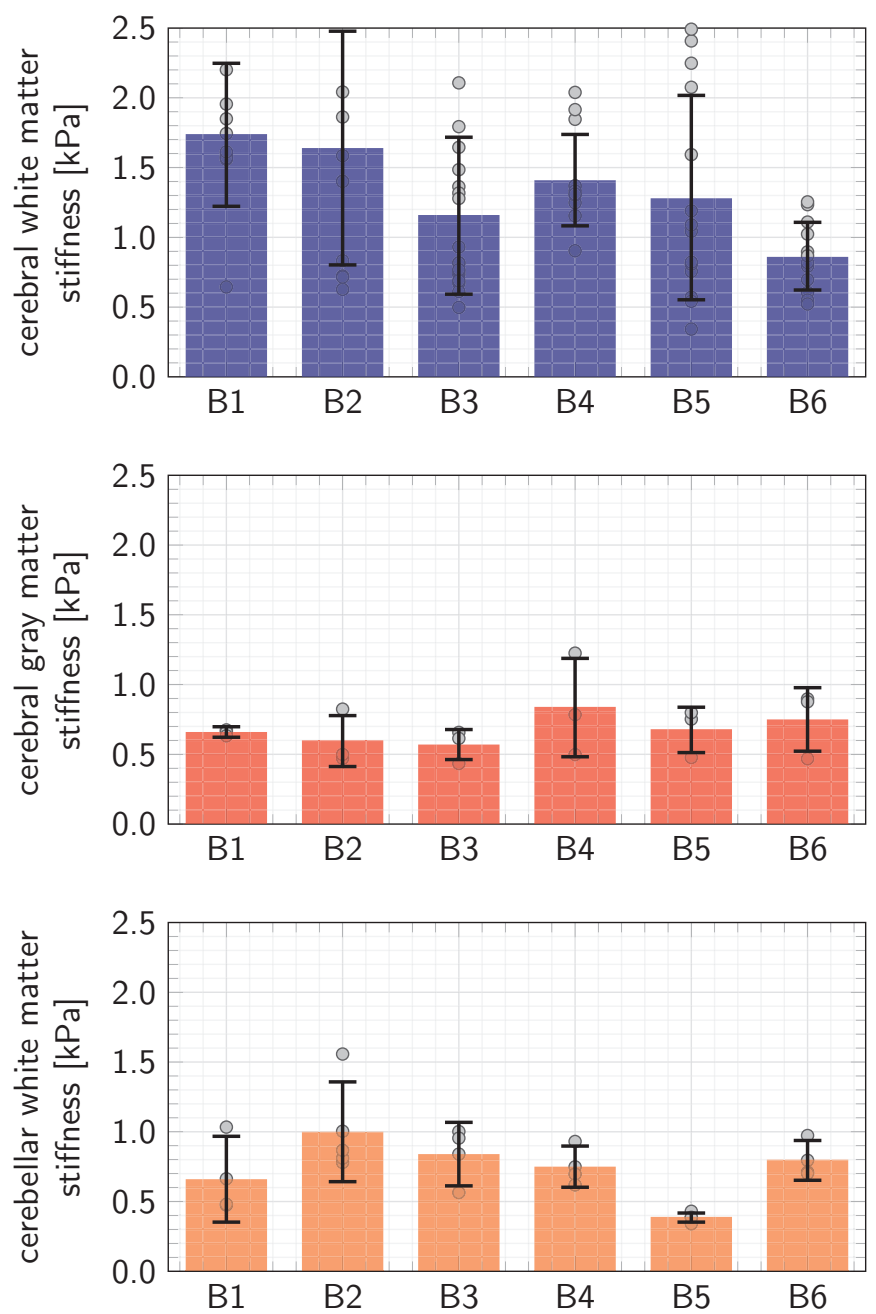

Figure 3: Stiffnesses of different tissue types in six brains. We tested a total of $n=80$ samples of cerebral white matter, $n=18$ samples of cerebral gray matter, and $n=18$ samples of cerebellar white matter. Individual measurements are indicated as dots; means and standard deviations are shown as bar graphs. 

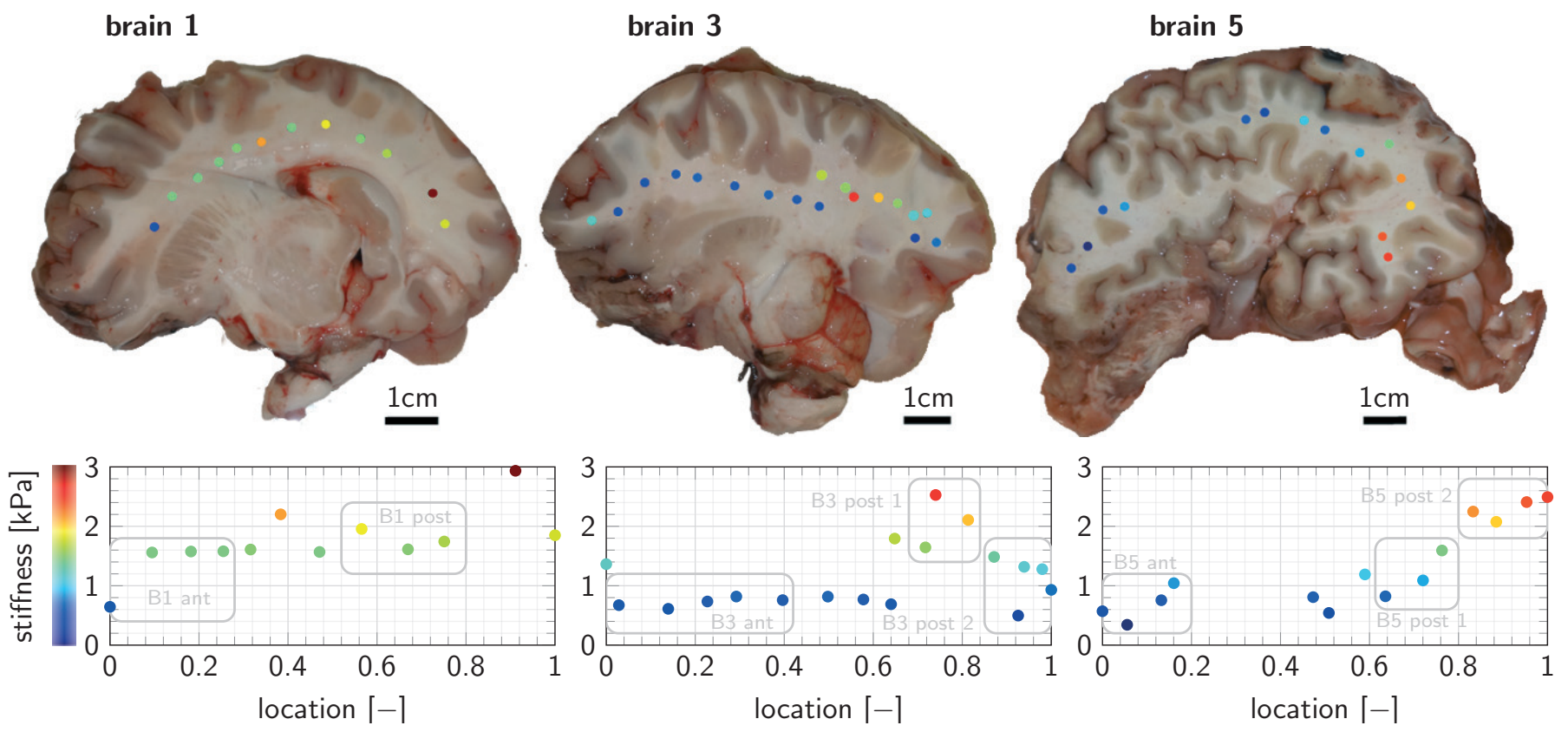

Figure 5: Stiffnesses of different regions of cerebral white matter. The cerebral white matter stiffnesses in the anterior and posterior regions across all six brains were significantly different $(\mathrm{p}<0.01)$. Stiffnesses ranged from $0.64 \mathrm{kPa}$ to $2.93 \mathrm{kPa}$ in brain 1 , from $0.50 \mathrm{kPa}$ to $2.53 \mathrm{kPa}$ in brain 3 , and from $0.34 \mathrm{kPa}$ to $2.49 \mathrm{kPa}$ in brain 5 . To explore to what extent these variations were correlated to the underlying tissue microstructure, we performed a histological characterization of eight samples, indicated through the gray boxes.

are indicated as dots; means and standard deviations are shown as bar graphs.

Figure 4 summarizes the stiffnesses in the white matter cerebrum, the gray matter cerebrum, and the white matter cerebellum across all six brains. We measured a cerebral white matter stiffness of $1.33 \pm 0.63 \mathrm{kPa}$, a cerebral gray matter stiffness of $0.68 \pm 0.20 \mathrm{kPa}$, and a cerebellar white matter stiffness of $0.75 \pm 0.29 \mathrm{kPa}$. The mean stiffness in cerebral white matter with $1.33 \mathrm{kPa}$ was almost twice as

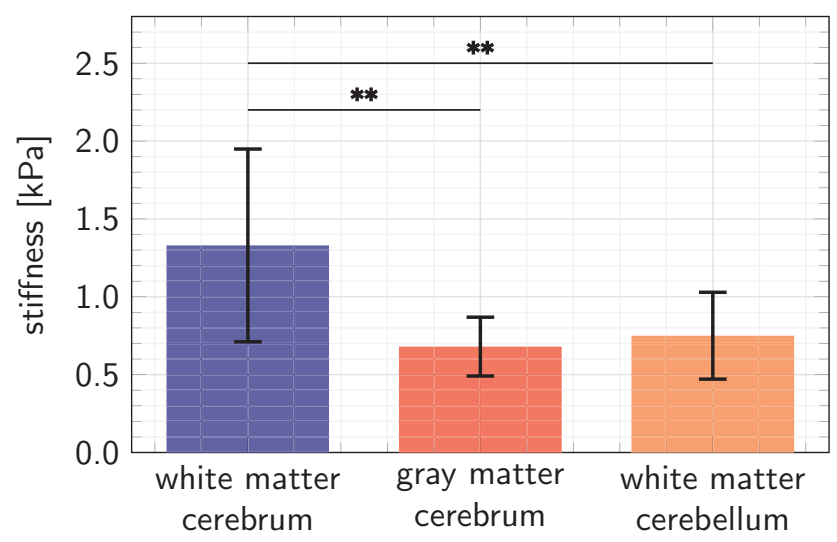

Figure 4: Stiffnesses of different tissue types in six brains. The cerebral white matter stiffness was $1.33 \pm 0.63 \mathrm{kPa}$, the cerebral gray matter stiffness was $0.68 \pm 0.20 \mathrm{kPa}$, and the cerebellar white matter stiffness was $0.75 \pm 0.29 \mathrm{kPa}$. An analysis of variance and a posthoc Tukey's test revealed that stiffness of cerebral white matter was significantly different from both cerebral gray matter and cerebellar white matter $(* * \mathrm{p}<0.01)$. large as in cerebral gray matter with $0.68 \mathrm{kPa}$ and cerebellar white matter with $0.75 \mathrm{kPa}$, which displayed comparable stiffnesses. An analysis of variance of the stiffness measurements in cerebral white matter, cerebral gray matter, and cerebellar white matter yielded a significant variation among the three tissue types, $\mathrm{F}=3.68(\mathrm{p}<0.01)$. A post-hoc Tukey's test revealed that the stiffness of cerebral white matter was significantly different from both cerebral gray matter and cerebellar white matter $(\mathrm{p}<0.01)$; stiffness values for cerebral and cerebellar white matter were not significantly different. Interestingly, the standard deviation in cerebral white matter with $47 \%$ was noticeably larger than in cerebral gray matter with $29 \%$ and in cerebellar white matter with $39 \%$.

Fig. 5 illustrates the stiffnesses of different cerebral white matter regions in three representative brains. The average stiffness displayed moderate inter-specimen variations. It varied from $1.74 \pm 0.53 \mathrm{kPa}$ for brain 1 , to $1.16 \pm 0.57 \mathrm{kPa}$ for brain 3 , and $1.28 \pm 0.74 \mathrm{kPa}$ for brain 5 . However, within each individual brain, the stiffness displayed marked regional variations along the anteriorposterior arch. The average minimum and maximum stiffnesses were $0.59 \pm 0.19 \mathrm{kPa}$ and $2.36 \pm 0.64 \mathrm{kPa}$. For the displayed brains, stiffness values ranged from $0.64 \mathrm{kPa}$ to $2.93 \mathrm{kPa}$ in brain 1 , from $0.50 \mathrm{kPa}$ to $2.53 \mathrm{kPa}$ in brain 3 , and from $0.34 \mathrm{kPa}$ to $2.49 \mathrm{kPa}$ in brain 5 . Brain 5 displayed the largest regional variation, with the smallest and largest stiffnesses varying by almost one order of magnitude. By clustering our cerebral white matter stiffness data across all six brains using partitioning around medoids, we observed a statistically significant stiffness 
difference in the anterior and posterior regions. In fact, a t-test confirmed $(\mathrm{p}<0.01)$ that the anterior stiffness of $0.95 \pm 0.32 \mathrm{kPa}$ was significantly lower than posterior stiffness of $2.04 \pm 0.45 \mathrm{kPa}$. From a macrostructural inspection of the anterior and posterior regions in Fig. 5, we observed no visible difference that could explain such a large stiffness variation. To test our hypothesis that this variation is correlated with the underlying tissue microstructure, we prepared eight representative regions, highlighted through the gray boxes in Fig. 5, for a thorough histological characterization.

\subsection{Histological characterization}

Fig. 6 shows representative histological stains of five different brain regions. The individual columns display the combined hematoxylin and eosin and luxol fast blue stain, left, the hematoxylin and eosin stain, middle, and the luxol fast blue stain, right. The individual rows illustrate the microstructural specificity at five different locations, at the gray and white matter interface in a gyrus (a) and in a sulfcus (b), in the cerebellar white matter (c), and in cerebral white matter with high (d) and low (e) myelin content. The percentage values in each stain indicate the red- and blue-colored area fractions, which we interpret as the regional protein and myelin contents. In Fig. 6 (a) and (b), at the gray and white matter interface, the myelin content is a global macrostructural indicator for the transition from gray to white matter. In Fig. 6 (d) and (e), in the cerebral white matter, the myelin content is a local microstructural indicator for the degree of myelination, which varies from $56 \%$ in Fig. 6 (e) to to $81 \%$ in Fig. $6(\mathrm{~d})$.

Fig. 7 illustrates the stiffness-myelin relation in the eight different regions of cerebral white matter indicated through the gray boxes in Fig. 5. The color-coded dots indicate the mean stiffness and mean myelin content in each region; the gray ellipses illustrate the associated standard deviations in stiffness and in myelin content. The cerebral white matter stiffness increased with increasing myelin content, from $0.72 \mathrm{kPa}$ at a myelin content of $64 \%$ to $2.45 \mathrm{kPa}$ at a myelin content of $89 \%$. Stiffness and myelin content were highly correlated with a Pearson correlation coefficient of $\rho=0.91(\mathrm{p}<0.01)$.

\section{Discussion}

\subsection{Mechanical characterization}

With an increasing recognition of the physical environment of the brain $[25,26]$, the mechanical characterization of gray and white matter tissue is receiving growing attention [1, 2]. Yet, the material properties reported in the literature continue to show variations of one order of magnitude and more [27]. At the same time, a direct comparison of the reported parameter values remains challenging because of differences in experimental methods, loading protocols, and sample preparation

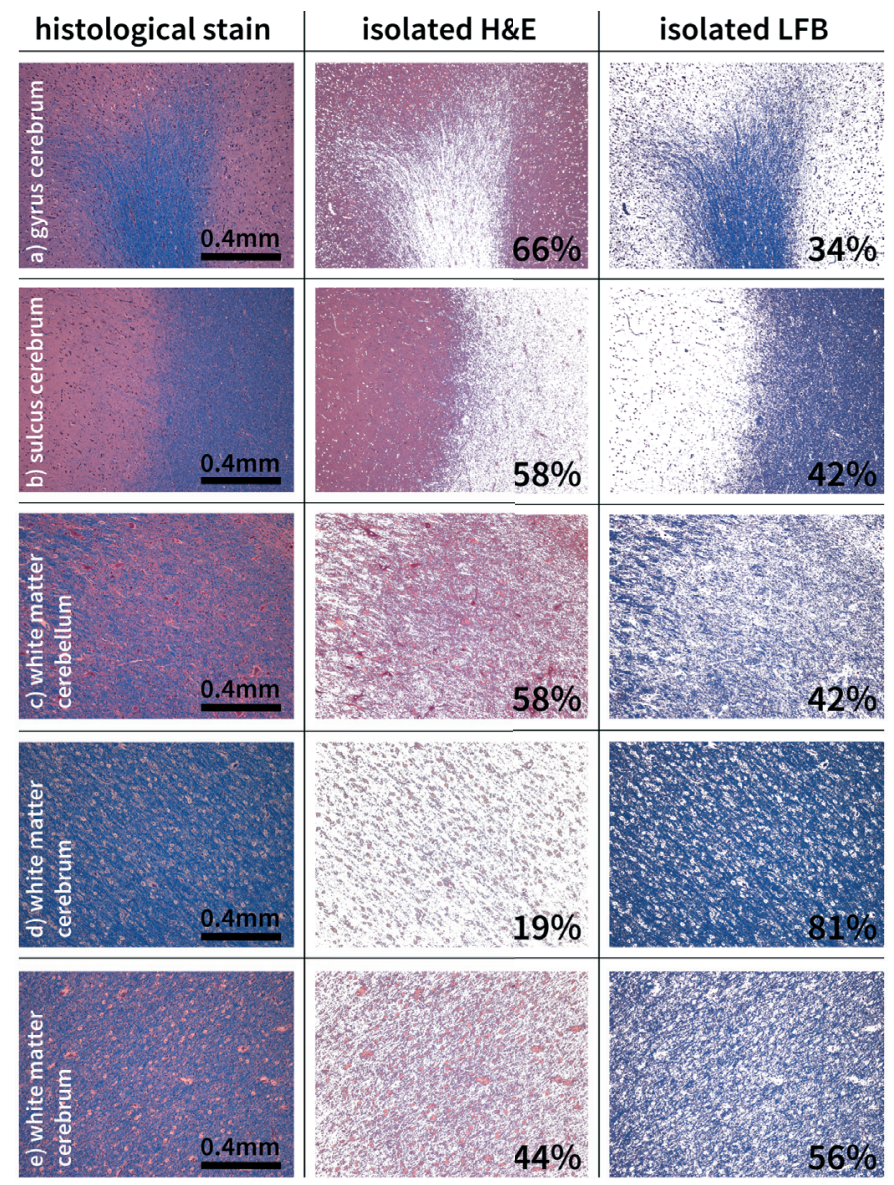

Figure 6: Histological stains of different brain regions. Combined hematoxylin and eosin (H\&E) and luxol fast blue (LFB) stain (left), isolated H\&E stain to highlight intracellular and extracellular proteins (middle), and isolated LFB stain to highlight myelin (right). Images show gray-and-white matter interface in a gyrus (a) and in a sulcus (b), cerebellar white matter (c), and cerebral white matter with high (d) and low (e) myelin content. Percentage values indicate the region-specific protein content, shown in red, and myelin content, shown in blue.

[28]. As an experimental method, we selected indentation testing and equipped a commercially available test set-up for nanoindentation with an extended displacement stage and a custom-designed $1.5 \mathrm{~mm}$ diameter flat-punch indenter. As we have perviously shown, this set-up allows us to probe freshly harvested fully intact tissue slices, reduce tissue degradation, and minimize sample dehydration [14]. As loading protocol, we selected a slow displacementcontrolled loading-holding-unloading profile, which we analyzed using a custom-designed, semi-automatic procedure. This approach successfully minimized tissue adhesion, which plays a critical role during ultrasoft matter indentation [29].

\subsection{Gray and white matter stiffnesses}

The current brain mechanics literature features an ongoing controversy about the stiffnesses of gray and white matter, with several studies reporting gray matter to be twice as stiff as white [16, 17], several finding reporting 


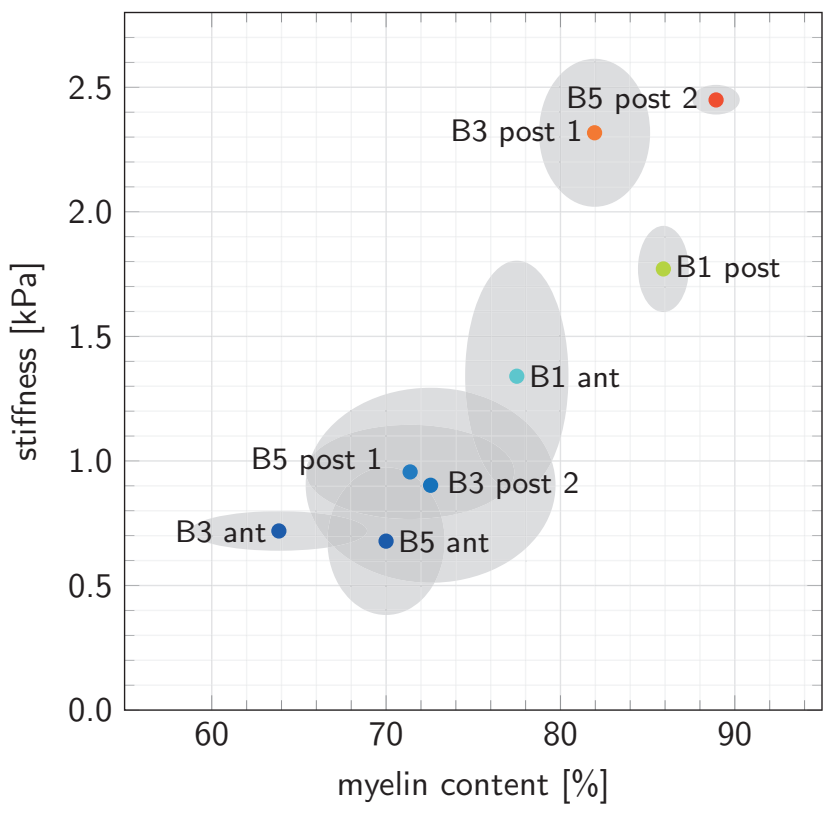

Figure 7: Stiffness-myelin relation in different regions of cerebral white matter. Color-coded dots indicate mean, gray ellipses indicate standard deviation in stiffness and myelin content. Stiffness increased with increasing myelin content, from $0.72 \mathrm{kPa}$ at a myelin content of $64 \%$ to $2.45 \mathrm{kPa}$ at a myelin content of $89 \%$. Stiffness and myelin content were highly correlated with a Pearson correlation coefficient of $\rho=0.91(\mathrm{p}<0.01)$.

gray matter to be one third softer than white matter [14, 12, 18], and several studies reporting no significant stiffness differences $[4,19]$. Knowing the precise stiffness ratio between gray and white matter is significant, since this ratio plays a critical role during cortical folding in human brain development [30, 31]. One objective of our study was therefore to explain the discrepancy between gray and white matter stiffness measurements. In $\mathrm{n}=116$ stiffness measurements highlighted in Fig. 3, we found that the cerebral white and gray matter stiffnesses were significantly different $(\mathrm{p}<0.01)$; cerebral white matter was on average twice as stiff as cerebral gray matter. Interestingly, white matter, with a standard deviation of $49 \%$, displayed a significantly larger variation than gray matter, with a standard deviation of $29 \%$. This observation agrees well with findings in the literature, which also reported white matter to be stiffer than gray, with a larger standard deviation [12]. This previous study attributed the large regional stiffness variation in white matter to its more anisotropic and heterogeneous microstructure, compared to the rather isotropic and homogeneous microstructure of gray matter [32, 33]. While gray matter consists primarily of neuronal cell bodies, dendrites, and unmyelinated axons, white matter is heterogeneously made up of myelinated axons and other cell types, oligodendrocytes, astrocytes, and microglia [34, 35]. Fig. 5 illustrates the large standard deviation in white matter stiffness in three representative brains. The average maximum white matter stiffness across all six brains was $2.36 \pm 0.64 \mathrm{kPa}$, which is four times larger than the average minimum white matter stiffness of $0.59 \pm 0.19 \mathrm{kPa}$. In each of the six analyzed brains, the smallest white matter stiffness was smaller than the brain's average gray matter stiffness, and the largest white matter stiffness was larger. These results suggest that, even when using the same experimental method, the same loading protocol, the same sample preparation, the same species, and the same brain, we cannot identify a single, unique stiffness value that characterizes the brain's stiffness. The brain's white matter stiffness displays tremendous regional variations. To better understand the mechanistic origin of these variations was the main focus of this study.

\subsection{Histological characterization}

Upon visual inspection of the samples in Fig. 5, we did not observe significant regional differences in white matter macrostructure that could explain our large stiffness variations. To analyze white matter microstructure, we performed combined hematoxylin and eosin and luxol fast blue staining [36]. Hematoxylin colors cell nuclei in blue and a subsequent counterstaining with eosin colors eosinophilic intracellular and extracellular protein structures in various shades of red, pink, and orange. Luxol fast blue is a sulfonated copper phthalocyanine dye that is attracted to the bases in the lipoproteins of the myelin sheath and colors them in blue whereas neuropil appears pink, and nerve cells appear purple [37]. This combined staining allowed us to macrostructurally differentiate regions of gray and white matter, as highlighted in Fig.6 (a) and (b), and to microstructurally quantify the myelin content of white matter tissue, as illustrated in Fig.6 (c), (d) and (e). Upon image analysis, we recorded regional variations from $56 \%$ to $81 \%$ in the local myelin content of the cerebral white matter tissue. While the luxol fast blue stain can quantify the variation in myelin content, it cannot answer the question whether this variation is caused by a variation in the degree of myelination, by a variation in the axonal density, or both.

\subsection{White matter stiffness increases with myelin content}

Our measurements in Fig. 5 indicate a trend towards white matter stiffening along the anterior-posterior arch. Yet, because of the current sample size, this regional stiffness gradient was not strong enough to be statistically significant. However, our observed trend agrees well with findings reported in the literature for bovine brain [38] and human brain [39]. In particular the latter study describes a density gradient in the white matter myelin content from a lower myelin content in the anterior region to a higher myelin content in the posterior region [39]. This also agrees with recent magnetic resonance elastography studies that report a stiffness gradient along the anterior-posterior arch in different substructures of the brain [40]. Although we did not observe a well-defined gradient in either stiffness or myelin content along the anterior-posterior arch, we found 
an explicit correlation between both parameters. Fig. 7 visualizes the relation between cerebral white matter stiffness and myelin content in the eight analyzed subregions highlighted through the gray boxes in Fig. 5. Our study suggests that the cerebral white matter stiffness and the local myelin content are highly correlated; in our case, with a Pearson correlation coefficient of $\rho=0.91(\mathrm{p}<0.01)$. These observations are in line with a recent study that reported a strong correlation between mechanical properties from atomic force microscopy and ultrastructure from immunohistochemistry [16].

\subsection{Clinical implications}

Our findings indicate a clear correlation between white matter stiffness and myelin content. This correlation could have significant clinical implications. During development, cortical folding occurs at a very low stiffness ratio between gray and white matter and takes place in parallel with the onset of myelination [34]. Several mathematical models have shown that the developing folding pattern is highly sensitive to the stiffness contrast between gray and white matter [41], and that even small changes in stiffness could have a large effect on the emerging brain surface morphology [30, 42, 43]. Dysmyelination, the delayed or premature formation of myelin sheaths, could induce pathological softening or stiffening, which could compromise cortical folding and normal brain development. Developmental white matter diseases [44] are associated with psychological disorders such as schizophrenia, autism, and epilepsy $[45,46]$. Other myelin-based disorders are known to cause cerebellar ataxia, spasticity, and optic atrophy [47]. A typical example is hypomyelination, vanishing white matter disease, which affects early myelinating structures in the brain. Our findings suggest that myelin-based alterations directly affect the stiffness of white matter tissue, and with it the mechanical environment of the brain. These changes directly impact our brain's elasticity, viscoelasticity [48], and poroelasticity [49], which, in turn, could affect extracellular matrix turnover and immune cell infiltration [50]. Taken together, our study indicates that myelin not only performs a critical function in controlling the electrical environment of our brain, but also plays an important role in modulating its mechanical environment.

\subsection{Limitations}

Our current study has a few limitations that could point towards directions for future work. First, the study was performed on bovine brain, and it remains to be shown to what extent the results translate to human brain tissue. Second, we have assumed that the blue area fraction of the luxol fast blue stain is directly related to the local myelin content, which could be overly simplistic. Moreover, the luxol fast blue stain alone cannot answer whether variations in myelin content are caused by variations in the degree of myelination, variations in the axonal density, or both. In addition, we have only characterized tissue microstructure by means of the local myelin content, and have neglected all other cell types including oligodendrocytes, astrocytes, and microglia. We are currently performing a systematic analysis of different histological stains to explore to what extent other microstructural elements vary across the brain and contribute to shaping the mechanical environment. Third, it would be interesting to analyze brains of different ages to characterize the chronological evolution of stiffness and microstructure in the developing brain, both in time and space. Last, our study characterizes the correlation between stiffness and myelin content ex vivo and it remains to be shown whether this correlation is equally present in the living brain in vivo. In its in vivo state, white matter in the intact brain has been reported to be under tension along the fiber directions [32]. This residual stress would affect the apparent modulus of white matter. It is plausible, even likely, that the degree of tension is different in different regions of the brain [30]. The original slicing of brain tissue into $5 \mathrm{~mm}$-thick slices is likely to relieve residual stress in a heterogeneous manner. While we expect our $5 \mathrm{~mm}$-thick slices to preserve the biological state better than 5mm-sized cubes, only true in vivo testing, e.g., through magnetic resonance elastography [51], will provide clarification to which extent the mechanical properties differ in live and dead brain tissue.

\section{Conclusion}

Although brain stiffness is broadly acknowledged to play an important role in neurophysiology and neuropathology, reliable stiffness measurements are sparse and reported values vary significantly. Little is known about how the microstructural architecture of our brain affects its macrostructural response. Here we used a hybrid mechanical-histological approach to establish a direct correlation between white matter stiffness and microstructure. Specifically, we demonstrated that the local stiffness of white matter tissue is highly correlated to the regional myelin content. In 116 indentation tests of six freshly harvested bovine brains, we found that cerebral white matter, with a stiffness of $1.33 \pm 0.63 \mathrm{kPa}$, was almost twice as stiff as cerebral gray matter, with $0.68 \pm 0.20 \mathrm{kPa}$, and also displayed a markedly larger regional variation. To explain this variation, we quantified the local myelin content using combined hematoxylin and eosin and luxol fast blue staining, and found that the white matter stiffness increased with increasing myelin content, from $0.5 \mathrm{kPa}$ at a myelin content of $63 \%$ to $2.5 \mathrm{kPa}$ at a myelin content of $92 \%$. Our results may have implications in neurodevelopment and neurological disease. In development, incomplete myelination could help explain-at least in part-why immature brains are softer than myelinated, mature brains and more vulnerable to mechanical insult, for example, in shaken baby syndrome. In disease, the degree of brain softening could serve as a clinical marker to quantify the degree of demyelination and characterize the onset of demyelinating disease, for example, in multiple sclerosis. Our study suggests that myelin not only plays an important function 
in insulating signal propagation and improving electrical function of single axons, but also in providing structural support and mechanical stiffness to the brain as a whole.

\section{Acknowledgements}

This study was supported by the German National Science Foundation grant STE 544/50-1 and by the Bavaria California Technology Center grant 'Mechanical Characterization of Brain Development' to Silvia Budday and Paul Steinmann and by the Bio-X IIP seed grant 'Understanding Gyrification Dynamics in the Human Brain' to Ellen Kuhl.

\section{References}

[1] K. Franze, P. A. Janmey, J. Guck, Mechanics in neuronal development and repair, Annual Review of Biomedical Engineering 15 (2013) 227-251.

[2] A. Goriely, M. G. Geers, G. A. Holzapfel, J. Jayamohan, A. Jérusalem, S. Sivaloganathan, W. Squier, J. A. W. van Dommelen, S. Waters, E. Kuhl, Mechanics of the brain: perspectives, challenges, and opportunities, Biomechanics and Modeling in Mechanobiology (2015) 1-35.

[3] U. Hamhaber, I. Sack, S. Papazoglou, J. Rump, D. Klatt, J. Braun, Three-dimensional analysis of shear wave propagation observed by in vivo magnetic resonance elastography of the brain, Acta Biomaterialia 3 (2007) 127-137.

[4] Y. Feng, R. J. Okamoto, R. Namani, G. M. Genin, P. V. Bayly, Measurements of mechanical anisotropy in brain tissue and implications for transversely isotropic material models of white matter, Journal of the Mechanical Behavior of Biomedical Materials 23 (2013) 117-132.

[5] K. J. Streitberger, I. Sack, D. Krefting, P. C., J. Braun, P. Friedemann, J. Wuerfel, Brain viscoelasticity alteration in chronic-progressive multiple sclerosis, PLoS ONE 7 (2012) e29888.

[6] I. Sack, B. Beierbach, J. Wuerfel, D. Klatt, U. Hamhaber, S. Papzpglou, P. Martus, J. Braun, The impact of aging and gender on brain viscoelasticity, NeuroImage 46 (2009) 652-657.

[7] A. Arvin, M. C. Murphy, K. J. Glaser, A. Manduca, D. S. Lake, S. A. Kruse, C. R. Jack, R. L. Ehman, J. Huston, Measuring the effects of aging and sex on regional brain stiffness with MRelastography in healthy older adults, NeuroImage 111 (2015) 59-64.

[8] R. Virchow, Ueber das ausgebreitete Vorkommen einer dem Nervenmark analogen Substanz in den thierischen Geweben, Virchows Archiv 6 (1854) 562-572.

[9] P. Morell, R. H. Quarles, Myelin formation, structure and biochemistry, in: G. J. Siegel, B. W. Agranoff, R. W. Albers, S. K. Fisher, M. D. Uhler (Eds.), Basic Neurochemistry: Molecular, Cellular and Medical Aspects, 6th ed., Lippincott-Raven, Philadelphia, 2003, pp. 69-94.

[10] L. J. Chew, P. Fusar-Poli, T. Schmitz, Oligodendroglial alternations and the role of microglia in white matter injury: relevance to schizophrenia, Developmental Neuroscience 35 (2013) 102129.

[11] C. Lucchinetti, W. Bruck, J. Parisi, B. Scheithauer, M. Rodriguez, H. Lassmann, Heterogeneity of multiple sclerosis lesions: implications for the pathogenesis of demyelination, Annals of Neurology 47 (2000) 707-717.

[12] J. A. W. van Dommelen, T. P. J. van der Sande, M. Hrapko, G. W. M. Peters, Mechanical properties of brain tissue by indentation: interregional variation, Journal of the Mechanical Behavior of Biomedical Materials 3 (2010) 158-166.
[13] T. P. Prevost, G. Jin, M. A. De Moya, H. B. Alam, S. Suresh, S. Socrate, Dynamic mechanical response of brain tissue in indentation in vivo, in situ and in vitro, Acta Biomaterialia 7 (2011) 4090-4101.

[14] S. Budday, R. Nay, R. de Rooij, P. Steinmann, T. Wyrobek, T. C. Ovaert, E. Kuhl, Mechanical properties of gray and white matter brain tissue by indentation, Journal of the Mechanical Behavior of Biomedical Materials 46 (2015) 318-330.

[15] D. MacManus, B. Pierrat, J. Murphy, M. Gilchrist, Dynamic mechanical properties of murine brain tissue using microindentation, Journal of Biomechanics 48 (2015) 3213-3218.

[16] D. E. Koser, E. Moeendarbary, J. Hanne, S. Kuerten, K. Franze, CNS cell distribution and axon orientation determine local spinal cord mechanical properties, Biophysical Journal 108 (2015) 2137-2147.

[17] A. F. Christ, K. Franze, H. Gautier, P. Moshayedi, J. Fawcett, R. J. Franklin, R. T. Karadottir, J. Guck, Mechanical difference between white and gray matter in the rat cerebellum measured by scanning force microscopy, Journal of Biomechanics 43 (2010) 2986 - 2992.

[18] T. Kaster, I. Sack, A. Samani, Measurement of the hyperelastic properties of ex vivo brain tissue slices, Journal of Biomechanics 44 (2011) 1158 - 1163.

[19] M. A. Green, L. E. Bilston, R. Sinkus, In vivo brain viscoelastic properties measured by magnetic resonance elastography, NMR in Biomedicine 21 (2008) 755-764.

[20] T. P. Prevost, A. Balakrishnan, S. Suresh, S. Socrate, Biomechanics of brain tissue, Acta Biomaterialia 7 (2011) 83-95.

[21] L. A. Mihai, L. K. Chin, P. A. Janmey, A. Goriely, A hyperelastic constitutive model for compression stiffening applicable to brain and fat tissues, Journal of the Royal Society Interface 12 (2015) 20150486.

[22] M. M. Blum, T. C. Ovaert, Experimental and numerical tribological studies of a boundary lubricant functionalized poroviscoelastic PVA hydrogel in normal contact and sliding, Journal of the Mechanical Behavior of Biomedical Materials 14 (2012) 248-258.

[23] M. M. Blum, T. C. Ovaert, A novel polyvinyl alcohol hydrogel functionalized with organic boundary lubricant for use as low?friction cartilage substitute: Synthesis, physical/chemical, mechanical, and friction characterization, Journal of Biomedical Materials Research B: Applied Biomaterials 100 (2012) 17551763.

[24] W. C. Oliver, G. M. Pharr, Measurement of hardness and elastic modulus by instrumented indentation: Advances in understanding and refinements to methodology, Journal of Materials Research 19 (2004) 3-20.

[25] A. Goriely, S. Budday, E. Kuhl, Neuromechanics: from neurons to brain, Advances in Applied Mechanics 48 (2015) 79-139.

[26] E. Kuhl, Biophysics: Unfolding the brain, Nature Physics (2016) in press; doi:10.1038/nphys3641.

[27] S. Chatelin, A. Constantinesco, R. Willinger, Fifty years of brain tissue mechanical testing: from in vitro to in vivo investigations, Biorheology 47 (2010) 255.

[28] K. Miller, Biomechanics of the Brain, Springer, New York, 2011.

[29] S. Gupta, F. Carrillo, C. Li, L. Pruitt, C. Puttlitz, Adhesive forces significantly affect elastic modulus determination of soft polymeric materials in nanoindentation, Materials Letters 61 (2007) $448-451$.

[30] P. V. Bayly, L. A. Taber, C. D. Kroenke, Mechanical forces in cerebral cortical folding: A review of measurements and models, Journal of the Mechanical Behavior of Biomedical Materials 29 (2014) 568-581.

[31] S. Budday, P. Steinmann, E. Kuhl, The role of mechanics during brain development, Journal of the Mechanics and Physics of Solids 72 (2014) 75-92.

[32] G. Xu, A. K. Knutsen, K. Dikranian, C. D. Kroenke, P. V. Bayly, L. A. Taber, Axons pull on the brain, but tension does not drive cortical folding, Journal of Biomechanical Engineering 132 (2010) 071013.

[33] C. Giordano, R. J. H. Cloots, J. A. W. van Dommelen, 
S. Kleiven, The influence of anisotropy on brain injury prediction, Journal of Biomechanics 47 (2014) 1052-1059.

[34] S. Budday, P. Steinmann, E. Kuhl, Physical biology of human brain development, Frontiers in Cellular Neuroscience 9 (2015).

[35] L. Bollmann, D. E. Koser, R. Shahapure, H. O. B. Gautier, G. A. Holzapfel, G. Scarcelli, M. C. Gather, E. Ulbricht, K. Franze, Microglia mechanics: immune activation alters traction forces and durotaxis, Frontiers in Cellular Neuroscience 9 (2014) 363.

[36] S. J. Oh, Color Atlas of Nerve Biopsy, CRC Press, Boca Raton, 2001.

[37] P. A. Humphrey, L. P. Dehner, J. D. Pfeifer, The Washington Manual of Surgical Pathology, Lippincott Williams \& Wilkins, Washington University, St. Louis, 2008.

[38] K. Urban, M. Hewicker-Trautwein, G. Trautwein, Development of myelination in the bovine fetal brain: an immunohistochemical study, Anatomia, Histologia, Embryologia 26 (1997) 187192.

[39] H. Braak, E. Ghebremedhin, U. Rüb, H. Bratzke, K. Del Tredici, Stages in the development of parkinsons disease-related pathology, Cell and Tissue Research 318 (2004) $121-134$.

[40] C. L. Johnson, M. D. McGarry, A. A. Gharibans, J. B. Weaver, K. D. Paulsen, H. Wang, W. C. Olivero, B. P. Sutton, J. G Georgiadis, Local mechanical properties of white matter structures in the human brain, Neuroimage 79 (2013) 145-152.

[41] S. Budday, E. Kuhl, J. W. Hutchinson, Period-doubling and period-tripling in growing bilayered systems, Philosophical Magazine (2015) 3208-3224.

[42] D. P. Richman, R. M. Stewart, J. Hutchinson, V. S. Cavincss Jr, Mechanical mode of brain convolutional deve lopment, Science 189 (1975) 18-21.

[43] S. Budday, C. Raybaud, E. Kuhl, A mechanical model predicts morphological abnormalities in the developing human brain, Scientific Reports 4 (2014) 5644.

[44] R. Schiffmann, M. S. van der Knaap, Invited article: an mribased approach to the diagnosis of white matter disorders, Neurology 72 (2009) 750-759.

[45] M. Gozzi, D. M. Nielson, R. K. Lenroot, J. L. Ostuni, D. A. Luckenbaugh, A. E. Thurm, J. N. Giedd, S. E. Swedo, A magnetization transfer imaging study of corpus callosum myelination in young children with autism, Biological Psychiatry 72 (2012) 215-220.

[46] M. Höistad, D. Segal, N. Takahashi, T. Sakurai, J. D. Buxbaum, P. R. Hof, Linking white and grey matter in schizophrenia: oligodendrocyte and neuron pathology in the prefrontal cortex, Frontiers in Neuroanatomy 3 (2009).

[47] P. J. Pouwels, A. Vanderver, G. Bernard, N. I. Wolf, S. F. Dreha-Kulczewksi, S. C. Deoni, E. Bertini, A. Kohlschütter, W. Richardson, W. Köhler, et al., Hypomyelinating leukodystrophies: translational research progress and prospects, Annals of Neurology 76 (2014) 5-19.

[48] R. de Rooij, E. Kuhl, Constitutive modeling of brain tissue: current perspectives, Applied Mechanics Reviews 68 (2016).

[49] G. Franceschini, D. Bigoni, P. Regitnig, G. A. Holzapfel, Brain tissue deforms similarly to filled elastomers and follows consolidation theory, Journal of the Mechanics and Physics of Solids 54 (2006) 2592-2620.

[50] K. Schregel, E. Wuerfel, P. Garteiser, I. Gemeinhardt, T. Prozorovski, O. Aktas, H. Merz, D. Petersen, J. Wuerfel, R. Sinkus, Demyelination reduces brain parenchymal stiffness quantified in vivo by magnetic resonance elastography, Proceedings of the National Academy of Sciences 109 (2012) 6650-6655.

[51] Y. Feng, E. H. Clayton, Y. Chang, R. J. Okamoto, P. V. Bayly, Viscoelastic properties of the ferret brain measured in vivo at multiple frequencies by magnetic resonance elastography, Journal of Biomechanics 46 (2013) 863-870. 